imatinib. Several mutant isoforms involving codons of exon 18 other than D842 showed sensitivity to the drug, however, as did those with exon 12 or exon 14 mutations, which affected the juxtamembrane domain and the first tyrosine kinase domain, respectively.

Pooling these results with those from previously published studies, the authors calculated that $63 \%$ of reported PDGFRA-mutant GISTs were likely to be resistant to imatinib. Since the remaining $37 \%$ of PDGFRA-mutant tumors might respond to this drug, however, they propose that molecular screening for KIT and PDGFRA mutations is a valid step in the management of GIST patients.

Ruth Kirby

Original article Corless CL et al. (2005) PDGFRA mutations in gastrointestinal stromal tumors: frequency, spectrum and in vitro sensitivity to imatinib. J Clin Oncol [doi: 10.1200/ JCO.2005.14.068]

\section{Optimal low-dose metronomic chemotherapy and antiangiogenic activity}

Results from an animal study have indicated that measuring the levels of circulating VEGFreceptor-2-positive endothelial progenitor cells, or CEPs, might help to determine the optimal biological dose (OBD) in patients undergoing metronomic chemotherapy.

Shaked and co-workers used four different mouse tumor models to assess both the efficacy and the toxicity of four metronomic chemotherapy regimens-cyclophosphamide, vinblastine, vinorelbine and cisplatinum. In each case, low doses of the drugs were administered at regular, short intervals with no prolonged breaks; this type of dosing is believed to maximise the antiangiogenic potential of the treatment. The OBD for each regimen was defined as the dose producing the greatest reduction in tumor volume, with the lowest toxicity. In paraIlel, the levels of viable CEPs in blood samples taken from the mice following one week of treatment were measured, and compared with those from untreated, control animals.

The analysis revealed a statistically significant, dose-dependent decrease in viable CEPs. For each treatment, the maximum fall in viable CEPs occurred in mice that had received the OBD of the drug, suggesting that the level of circulating CEPs might be a useful indicator of antiangiogenic activity.

The authors discuss the feasibility of translating these findings to the clinical setting, and conclude that "CEPs may serve as a pharmacodynamic biomarker to determine the optimal biological dose of metronomic chemotherapy regimens".

Ruth Kirby

Original article Shaked Y et al. (2005) The optimal biological dose of metronomic chemotherapy regimens is associated with maximum antiangiogenic activity. Blood [doi: 10.1182/blood-2005-04-1422]

\section{Preoperative anemia predicts poor outcome in surgically resected early-stage NSCLC patients}

A recently published retrospective analysis by a group at the University of Maryland School of Medicine has shown that preoperative anemia is a significant prognostic factor in patients with early-stage non-small-cell lung cancer (NSCLC) treated with surgery alone.

Relapse-free survival (RFS) and overall survival (OS) were calculated for 82 patients who had undergone surgical resection for stage I $(n=72)$ or II $(n=10)$ NSCLC. The 2-year RFS and OS for the entire cohort were $66 \%$ and $76.3 \%$, respectively. Preoperative hemoglobin $\mathrm{(Hb)}$ level was the only factor found to significantly influence outcome in both the univariate and multivariate analyses, although there was a nonsignificant trend toward reduced survival in p53-negative patients. Multivariate analysis revealed that patients with preoperative $\mathrm{Hb}$ levels $<12 \mathrm{mg} / \mathrm{dl}$ had significantly worse RFS and OS than those with $\mathrm{Hb}$ levels $\geq 12 \mathrm{mg} / \mathrm{dl}$ $(P=0.020$ and $P=0.026$, respectively).

The authors conclude that preoperative anemia is associated with increased risk of treatment failure and reduced survival, even in patients with traditionally low-risk, early-stage NSCLC. They suggest that anemia might be an indication of a more aggressive tumor and advocate further research into the prospect of using adjuvant therapy in these patients.

Alexandra King

Original article Yovino S et al. (2005) An association between preoperative anemia and decreased survival in earlystage non-small-cell lung cancer patients treated with surgery alone. Int J Radiat Oncol Biol Phys 62: 1438-1443 